\title{
Brain Tumours and Prophylactic Antiepileptic Drug Prescribing Patterns by Neurosurgeons Practising in Australasia
}

\author{
Chrisovalantis A. Tsimiklis*, Marguerite A. Harding \\ Royal Adelaide Hospital, North Terrace, Adelaide, Australia \\ Email: ctsimiklis29@gmail.com
}

Received 14 February 2015; accepted 1 March 2015; published 5 March 2015

Copyright (C) 2015 by authors and Scientific Research Publishing Inc.

This work is licensed under the Creative Commons Attribution International License (CC BY).

http://creativecommons.org/licenses/by/4.0/

\section{cC) (i) Open Access}

\begin{abstract}
Prescribing patterns amongst practising Australasian neurosurgeons regarding the use of prophylactic antiepileptic drugs (AEDs) in patients with newly diagnosed intrinsic brain tumours are not well established. This study aimed to determine the rate of prophylactic AED prescribing in this clinical context and to determine if there were some particular factors that influenced prescribers in their decision. A survey was conducted, and of the 91 respondents, $23(25.3 \%)$ prescribed prophylactic AEDs. No neurosurgeons practising in New Zealand prescribed, whereas within Australian states/territories, prescribing was most common in Western Australia $(3 / 4,75.0 \%)$ and Queensland $(8 / 18,44.4 \%)$ and less common in the Australian Capital Territory $(0 / 2,0.0 \%)$ and South Australia $(1 / 7,14.3 \%)$. The most commonly prescribed first-line AED was phenytoin (n $=15,68.2 \%)$ followed by levetiracetam $(n=5,22.7 \%)$. The duration of prescription varied from 1 week to 6 months, with 6 weeks chosen by most prescribers $(n=7,35 \%)$. Important factors that influence the decision to prescribe include tumour location and a history of previous seizure/s, whereas the presence of oedema or haemorrhage and patients' age do not seem to be major influences amongst prescribers.
\end{abstract}

\section{Keywords}

Brain Tumours, Antiepileptic Drugs, Seizure Prophylaxis, Medication Prescribing

\section{Introduction}

Patients with brain tumours, both primary and metastatic, are at an increased risk of experiencing seizures. The

Corresponding author.

How to cite this paper: Tsimiklis, C.A. and Harding, M.A. (2015) Brain Tumours and Prophylactic Antiepileptic Drug Prescribing Patterns by Neurosurgeons Practising in Australasia. Neuroscience \& Medicine, 6, 13-19.

http://dx.doi.org/10.4236/nm.2015.61003 
incidence of seizures amongst patients diagnosed with a brain tumour varies depending on certain factors, including tumour type and location. In a series of 1028 patients diagnosed with primary brain tumours, those with low-grade gliomas had a higher prevalence of epilepsy (85\%) compared with patients with a diagnosis of glioblastoma (49\%) [1]. Seizures are less common in metastases, reported in $24 \%$ in one series [2]. However, this is also dependent on the type of metastasis, with melanoma seemingly the most epileptogenic (67\%), reflective of a tendency to haemorrhage and to involve the cerebral cortex.

Epilepsy can be a significant burden on the patient due to the physical and psychosocial effects of both the condition and the treatment required. Although there is strong evidence against the use of AEDs for seizure prophylaxis in patients with brain tumours, they are certainly still prescribed in this clinical context. Overall, the adverse effects of AED therapy, including side effects and drug interactions, outweigh the benefits gained in preventing seizures in this patient group.

Data on physician practice in Rhode Island demonstrated that prophylactic anticonvulsant administration was $55 \%$ in a cohort of 115 [3]. There were differences between specialties with $33 \%$ of radiation oncologists prescribing, compared with $81 \%$ of neurosurgeons. There are limited data on current practice in Australasia and so here we aim to establish the prescribing patterns amongst Australasian neurosurgeons and to determine if there are some particular factors that influence the decision to be a prescriber.

\section{Methods}

\subsection{Study Approval}

Approval for this study was obtained from the Research Ethics Committee of the Royal Adelaide Hospital (HREC/14/RAH/59) and the Board of the Neurosurgical Society of Australasia (NSA) in line with the Requests to Conduct Research Policy.

\subsection{Study Design}

A simple survey was conducted targeting current practising Australasian neurosurgeons who are members of the NSA. The survey consisted of five questions designed to establish the general prescribing patterns when treating patients with newly diagnosed intrinsic brain tumours, in relation to seizure prophylaxis. This excluded treatment of extra-axial lesions (such as meningiomas) and other neurosurgical conditions. After approval, the survey was created using Survey Monkey ${ }^{\circledR}$ and NSA members were invited via email to respond to the survey.

\subsection{Survey Questions}

The survey consisted of five questions, four of which were selected for final analysis (Table 1). One question was omitted from the final analysis as it was similar to question 4 and thus had a low response rate $(13 / 23$, $56.5 \%$ ). Those who answered, "Yes", to the first question could proceed to the remaining questions. Those who answered, "No", could submit the survey after the first question.

\subsection{Data Analysis}

The data was entered into a Microsoft Excel ${ }^{\circledR}$ spreadsheet and tables/graphs were generated to analyse the information. The answer to question 1 was stratified to assess prescribing patterns based on country and state/territory of practice, as well as experience of the neurosurgeon (defined by decade of NSA admission).

\section{Table 1. Survey questions included in the final analysis.}

\section{Survey questions}

\footnotetext{
1) Do you routinely prescribe AEDs for prophylaxis in patients with a newly diagnosed intrinsic brain tumour? [Options: yes; no]

2) Starting with the most frequent, number your commonly prescribed first-line AEDs [Options: phenytoin; levetiracetam;

sodium valproate; lamotrigine; carbamazepine]

3) What is your duration of prescription? (answer to the closest duration) [Options: 1 week; 6 weeks; 3 months; 6 months; indefinitely]

4) Which of the following factors influence your decision on prescribing prophylactic AEDs in a patient with a newly diagnosed intrinsic brain tumour? [Options: tumour location; previous seizures; presence of oedema; presence of haemorrhage; patient's age]
} 


\section{Results}

A total of 91 responses were received from the 141 NSA members to whom the survey was distributed (64.5\% response rate). Of these, 80 and 11 were neurosurgeons currently practising in Australia and New Zealand (NZ), respectively. The majority of the Australian responders were from New South Wales (NSW) [ $n=26]$, Victoria (VIC) [ $\mathrm{n}=23]$ and Queensland (QLD) [n = 18], with fewer contributions from the remaining states or territories. No responses were received from Tasmanian (TAS) neurosurgeons. The Northern Territory (NT) was not included in the analysis as there is no neurosurgical unit based there. Experience was stratified by decade of admission to the NSA, noting the earliest respondent practising since 1971.

\subsection{Prophylactic AED Use}

Of the 91 respondents, 23 (25.3\%) routinely prescribe AEDs for prophylaxis in patients with a newly diagnosed intrinsic brain tumour (Figure 1). No neurosurgeons practising in NZ prescribe, whereas 23 out of the 80 (28.8\%) Australian respondents do. When stratified by state/territory, neurosurgeons practising in the Australian Capital Territory (ACT) and South Australia (SA) were less inclined to prescribe, $0(0 \%)$ and $1(14.3 \%)$, respectively. The greatest rates of prescribing were in Western Australia (WA) and QLD where a total of 3 (75\%) and 8 (44.4\%) neurosurgeons, respectively, prescribe prophylactic AEDs. Stratification by neurosurgeon experience showed a relatively constant prescribing pattern $(23.3 \%-25.0 \%)$ with the exception being the decade between 1981 and 1990, where $3(50 \%)$ neurosurgeons prescribe AEDs in this clinical context. The aforementioned data is summarised in Table 2, and Figure 2 and Figure 3 are column charts that provide a visual representation of the data stratified by state/territory and experience of neurosurgeon, respectively.

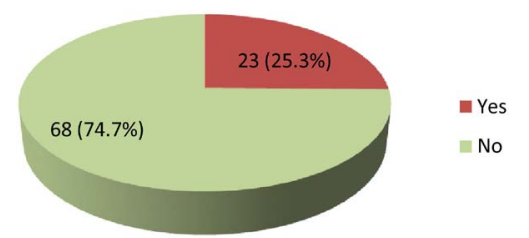

Figure 1. Pie chart demonstrating the distribution of prescribers vs. non-presecribers of prophylactic AEDs in patients with a newly diagnosed intrinsic brain tumour.

Table 2. Prescribing patterns stratified by country and state/territory of practice \& experience of neurosurgeon.

\begin{tabular}{|c|c|c|c|}
\hline Stratified variable & Total number & Prescribers & Percentage \\
\hline & $(\mathrm{n}=91)$ & $(\mathrm{n}=23)$ & $(\%)$ \\
\hline \multicolumn{4}{|l|}{ Country } \\
\hline Australia & 80 & 23 & 28.8 \\
\hline New Zealand & 11 & 0 & 0.0 \\
\hline \multicolumn{4}{|l|}{ State } \\
\hline NSW & 26 & 5 & 19.2 \\
\hline VIC & 23 & 6 & 26.1 \\
\hline QLD & 18 & 8 & 44.4 \\
\hline SA & 7 & 1 & 14.3 \\
\hline WA & 4 & 3 & 75.0 \\
\hline $\mathrm{ACT}$ & 2 & 0 & 0.0 \\
\hline TAS & 0 & 0 & 0.0 \\
\hline \multicolumn{4}{|c|}{ Experience of neurosurgeon ${ }^{*}$} \\
\hline $1971-1980$ & 12 & 3 & 25.0 \\
\hline $1981-1990$ & 6 & 3 & 50.0 \\
\hline $1991-2000$ & 30 & 7 & 23.3 \\
\hline 2001-2010 & 30 & 7 & 23.3 \\
\hline 2011-present & 13 & 3 & 23.1 \\
\hline
\end{tabular}

\footnotetext{
"Based on decade of admission to the NSA.
} 


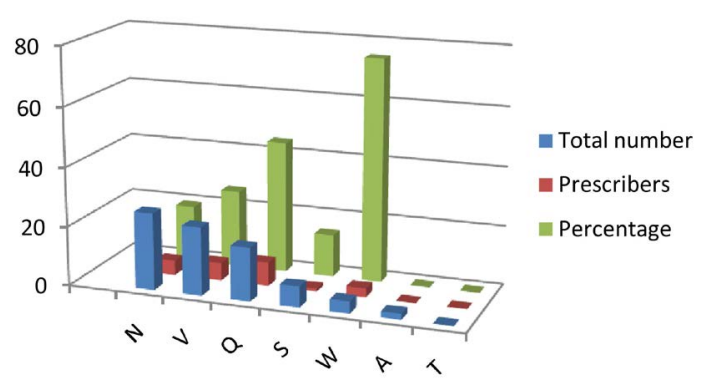

Figure 2. Column chart representing the distribution of prescribers of prophylactic AEDs in patients with a newly diagnosed intrinsic brain tumour, stratified by state/territory.

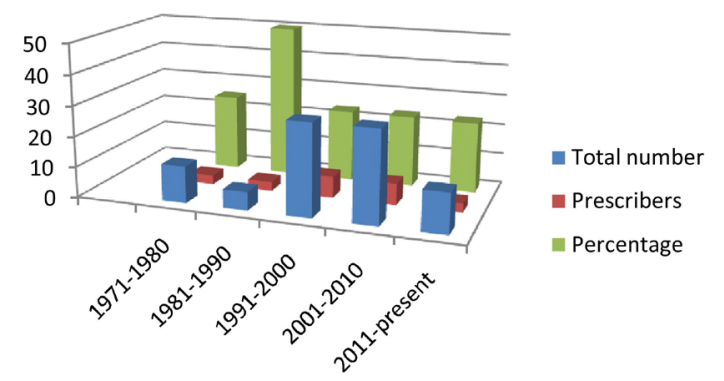

Figure 3. Column chart representing the distribution of prescribers of prophylactic AEDs in patients with a newly diagnosed intrinsic brain tumour, stratified by experience of neurosugeon.

\subsection{First-Line AEDs}

The most popular first-line AED was phenytoin, prescribed by 15 of the $22(68.2 \%)$ responders (Figure 4). Levetiracetam was the first-line AED of choice for $5(22.7 \%)$ individuals, followed by sodium valproate $(\mathrm{n}=2$, 9.1\%). No prescribers choose lamotrigine or carbamazepine as first-line agents for seizure prophylaxis. One prescriber did not respond to this question.

\subsection{Duration of Prescribing}

There was a fairly even distribution of prescribing duration with $6(30.0 \%), 7(35.0 \%)$ and $5(25.0 \%)$ respondents prescribing prophylactic AEDs for 1 week, 6 weeks and 3 months, respectively (Figure 5). Only 2 (10.0\%) individuals prescribed for 6 months. No one prescribed prophylactic AEDs indefinitely. Three prescribers did not respond to this question.

\subsection{Factors That Influence the Decision to Prescribe AEDs}

Tumour location and history of previous seizure/s were common factors influencing the decision to prescribe AEDs with 19 (95.0\%) and $16(80.0 \%)$ of prescribers, respectively, selecting these options (Figure 6). The presence of oedema was an influencing factor for $7(35.0 \%)$ individuals. The presence of haemorrhage $(n=3,15.0 \%)$ and the age of the patient $(n=3,15.0 \%)$, did not appear to be major influencing factors. Three prescribers did not respond to this question.

\section{Discussion}

Seizures can be a major burden on a patient with potentially significant quality of life and lifestyle implications. In patients with a brain tumour who have suffered a seizure/s, there is no controversy regarding the use of AEDs to prevent future events. However, the prophylactic use of AEDs in patients with a newly diagnosed brain tumour, who have not had a seizure, remains an issue of debate and practice varies. This may reflect lack of clarity in the available literature. 


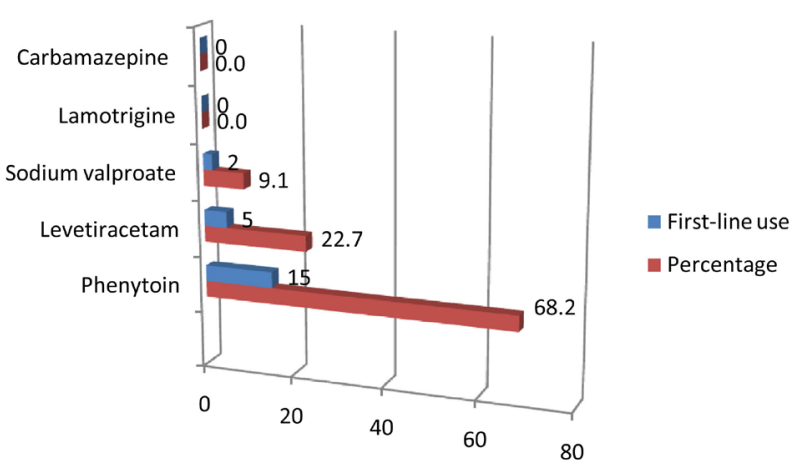

Figure 4. Bar chart demonstrating the distribution of first-line prophylactic AEDs prescribed in patients with a newly diagnosed intrinsic brain tumour.

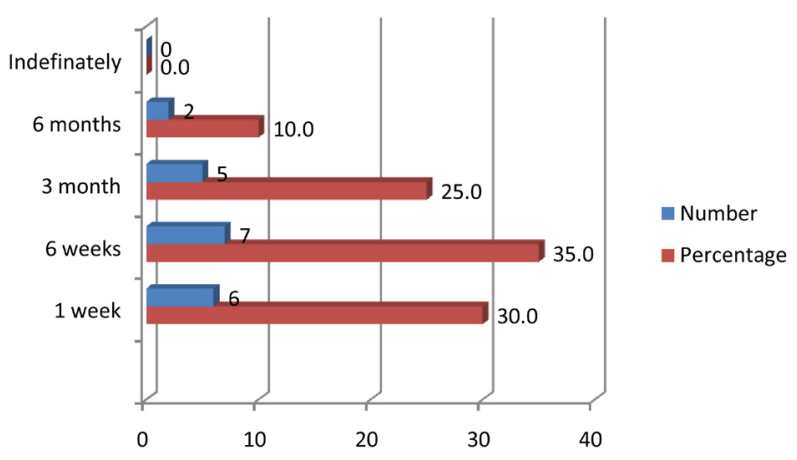

Figure 5. Bar chart demonstrating the duration of prescribing of prophylactic AEDs in patients with a newly diagnosed intrinsic brain tumour.

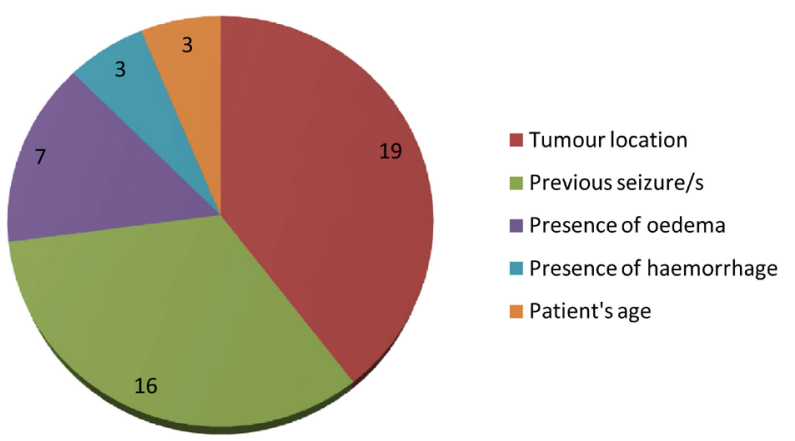

Figure 6. Pie chart demonstrating the factors that influence the decision to prescribe AEDs in patients with a newly diagnosed intrinsic brain tumour.

A meta-analysis of four randomised controlled trials (RCTs) and eight observational studies, found that prophylactic use of AEDs (specifically phenytoin, phenobarbital and divalproex sodium) is not effective in preventing first-onset seizures nor in improving the seizure-free and overall survival in patients with brain tumours [3]. In another meta-analysis of five RCTs that evaluated the efficacy of AED prophylaxis vs. no treatment or placebo to prevent seizures in patients with brain tumours who had no history of epilepsy, the conclusion was that there was no evidence to support their use regardless of neoplastic type [4].

Given that these patients will often proceed to a craniotomy, it is also important to consider the potential for seizures in the perioperative period. The question of perioperative seizure prophylaxis in patients with intrinsic brain tumours was explored in a RCT with a cohort of 123 patients, 62 of which were randomised to receive a 7- 
day postoperative phenytoin course (prophylaxis group) and 61 receiving no prophylaxis (observation group) [5]. They found that the incidence of early postoperative seizures (defined as seizures within 30 days of surgery) was $8 \%$ in the observation group compared with $10 \%$ in the prophylaxis group $(\mathrm{p}=1.0)$. The incidence of clinically significant early seizures was $3 \%$ and $2 \%$ in the observation and prophylaxis groups, respectively $(p=0.62)$. In addition the prophylaxis group experienced significantly more adverse events ( $18 \%$ vs. $0 \%, \mathrm{p}<0.01)$. The study concluded that the low baseline rate of perioperative seizures in patients with brain tumours raises concerns about the routine use of prophylactic phenytoin in this patient population.

Results of this survey indicate that prophylactic AED prescribing in this clinical context is not uncommon in Australasia despite the evidence, with a $25.3 \%$ rate of prescription. It was interesting to find that of the 11 respondents from NZ, none of them prescribe prophylactic AEDs. Whether or not this is due to specific guidelines in NZ is uncertain. Practising neurosurgeons in WA tended towards prescribing (75.0\%), noting that there were only 4 respondents from WA. Neurosurgeons in SA and ACT were less inclined to prescribe prophylactic AEDs. It was also interesting to note that there weren't any major differences in prescription rates based on neurosurgeon experience. The obvious exception was the decade between 1981 and 1990 where there was a 50.0\% prescription rate, although there were only 6 respondents from this era.

The main concern regarding prescription of AEDs relates to their potential adverse effects including side effects and drug interactions. AEDs can be associated with cognitive side effects, which impair patients who are already often cognitively affected by their condition and associated adjuvant treatments [6]. Although phenytoin has traditionally been the AED of choice amongst neurosurgeons, including in this study, it is a drug not without potential hazards. In one study it was found that patients on phenytoin, who are undergoing radiotherapy and weaning steroids, a common clinical scenario in patients with brain tumours, have an increased risk of erythema multiforme and Stevens-Johnson syndrome [7]. Phenytoin has a narrow therapeutic index and thus regular monitoring is required to avoid toxicity.

Levetiracetam is becoming a popular alternative AED drug of choice given that it is generally well tolerated by patients. In addition, unlike phenytoin, monitoring of plasma levetiracetam levels is not necessary and it is therefore easier to administer with dose adjustments made based on clinical response. Interestingly, presently under the general schedule of the Australian Government Department of Health's Pharmaceutical Benefits Scheme (PBS), levetiracetam is authorised only for the treatment of partial epileptic seizures which are not controlled satisfactorily by other anti-epileptic drugs [8]. This clearly precludes it from use as a prophylactic medication, unless prescribed as a non-PBS medication, which has potential cost implications for patients. Although seemingly safer than phenytoin, levetiracetam can cause side effects also with mood disturbance/irritability reported in approximately $19 \%$ of patients taking the drug [9].

Aside from side effects, the other major concern with the use of AEDs is the potential for drug-drug interactions. Through induction of the cytochrome P450 enzyme system, phenytoin and other prescribed AEDs can alter the metabolism of chemotherapeutic agents and steroids, reducing their effectiveness [10]. Conversely, the chemotherapeutic agents can induce metabolising enzymes, altering serum levels of AEDs [10].

Here we found that of those who prescribe, $68.2 \%$ choose phenytoin as a first-line prophylactic agent. Levetiracetam was the next most commonly prescribed first-line agent $(22.7 \%)$. Interestingly although sodium valproate can be associated with thrombocytopenia and it impairs platelet aggregation and thus can potentiate surgical bleeding [11], it was still prescribed by $9.1 \%$ of respondents as a first-line agent. On the other hand, there is some suggestion that patients with a diagnosis of glioblastoma who take sodium valproate, have a significantly longer survival when compared with patients not on AEDs or treated with other AEDs [12]. The proposed mechanism is related to its activity as a histone deacetylase inhibitor (HDI), with potential antitumour effect.

Through this simple survey, we have been able to explore the general prescribing patterns amongst Australasian neurosurgeons with regards to prophylactic AEDs. However, there are some limitations of this study that warrant discussion. Firstly, although there was a good overall response rate $(64.5 \%)$, without answers from the entire target group, there is obvious potential for bias. Also neurosurgeons who are not NSA members did not receive the invitation email. Amongst prescribers, there were several non-responders to the remaining questions. One of the questions was omitted completely from the final analysis due to a poor response rate. Furthermore, the style of the survey questions were closed and as such, further analysis is difficult. For example, although tumour location was selected as an important influencing factor by $95.0 \%$ of prescribers, there was no field to specify which particular locations are important. Overall however, the study was designed to gauge general prescribing patterns amongst Australasian neurosurgeons and to hopefully stimulate further debate regarding this 
topic.

\section{Conclusion}

Although the available evidence does not support the use of AEDs for preventing seizures in patients with brain tumours, clearly it is still commonplace amongst practising neurosurgeons, at least in Australia. Phenytoin remains the drug of choice, although levetiracetam is gaining popularity given its favourable side-effect profile and easier management. In terms of duration, 6 weeks was most commonly reported. The tumour location and a history of previous seizure(s) were the most important factors in the decision of prescribers. It will be interesting to see if current trends continue, and a follow-up survey will be worthwhile in the future.

\section{Conflict of Interest/Disclosure}

The authors declare that they have no financial or other conflicts of interest in relation to this research and its publication.

\section{References}

[1] Lote, K., Stenwig, A.E., Skullerud, K. and Hirschberg, H. (1998) Prevalence and Prognostic Significance of Epilepsy in Patients with Gliomas. European Journal of Cancer, 34, 98-102.

[2] Oberndorfer, S., Schmal, T., Lahrmann, H., Urbanits, S., Lindner, K. and Grisold, W. (2002) The Frequency Of seizures in Patients with Primary Brain Tumours or Cerebral Metastases. An Evaluation from the Ludwig Boltzmann Institute of Neuro-Oncology and the Department of Neurology, Kaiser Franz Josef Hospital, Vienna. Wien Klin Wochenschr, 114, 911-916.

[3] Glantz, M.J., Cole, B.F., Forsyth, P.A., et al. (2000) Practice Parameter: Anticonvulsant Prophylaxis in Patients with Newly Diagnosed Brain Tumors. Report of the Quality Standards Subcommittee of the American Academy of Neurology. Neurology, 54, 1886-1893. http://dx.doi.org/10.1212/WNL.54.10.1886

[4] Sirven, J.I., Wingerchuk, D.M., Drazkowski, J.F., Lyons, M.K. and Zimmerman, R.S. (2004) Seizure Prophylaxis in Patients with Brain Tumors: A Meta-Analysis. Mayo Clinic Proceedings, 79, 1489-94. http://dx.doi.org/10.4065/79.12.1489

[5] Wu, A.S., Trinh, V.T., Suki, D., et al. (2013) A Prospective Randomized Trial of Perioperative Seizure Prophylaxis in Patients with Intraparenchymal Brain Tumors. Journal of Neurosurgery, 118, 873-883. http://dx.doi.org/10.3171/2012.12.JNS111970

[6] Klein, M., Engelberts, N.H., van der Ploeg, H.M., et al. (2003) Epilepsy in Low-Grade Gliomas: The Impact on Cognitive Function and Quality of Life. Annals of Neurology, 54, 514-520. http://dx.doi.org/10.1002/ana.10712

[7] Khafaga, Y.M., Jamshed, A., Allam, A.A., et al. (1999) Stevens-Johnson Syndrome in Patients on Phenytoin and Cranial Radiotherapy. Acta Oncologica, 38, 111-116. http://dx.doi.org/10.1080/028418699431898

[8] Australian Government Department of Health [Internet] The Pharmaceutical Benefits Scheme (PBS). Australia. http://www.pbs.gov.au/medicine/item/8654L-8655M-8656N-9169N.

[9] Fuller, K.L., Wang, Y.Y., Cook, M.J., Murphy, M.A. and D’Souza, W.J. (2013) Tolerability, Safety, and Side Effects of Levetiracetam versus Phenytoin in Intravenous and Total Prophylactic Regimen among Craniotomy Patients: A Prospective Randomized Study. Epilepsia, 54, 45-57. http://dx.doi.org/10.1111/j.1528-1167.2012.03563.x

[10] Van Breemen, M.S., Wilms, E.B. and Vecht, C.J. (2007) Epilepsy in Patients with Brain Tumours: Epidemiology, Mechanisms, and Management. The Lancet Neurology, 6, 421-30. http://dx.doi.org/10.1016/S1474-4422(07)70103-5

[11] Page, M. (2007) Psychotropic Drugs and Dentistry. Australian Prescriber, 30, 98-101.

[12] Guthrie, G.D. and Eljamel, S. (2013) Impact of Particular Antiepileptic Drugs on the Survival of Patients with Glioblastoma Multiforme. Journal of Neurosurgery, 118, 859-65. http://dx.doi.org/10.3171/2012.10.JNS12169 